\title{
Parâmetros físico-químicos e biológicos de águas de tanques de estabilização
}

\author{
Physico-chemical and microbiological parameters of \\ water from stabilization ponds
}

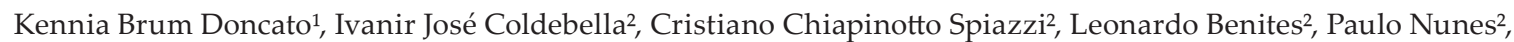
Thaís Mazzini², Priscila Trindade², Alessandra Tamajusuku Neis²

${ }^{1}$ Mestranda em Aquicultura, Universidade Federal do Rio Grande - FURG.

${ }^{2}$ Universidade Federal do Pampa - UNIPAMPA, Campus Uruguaiana

\begin{abstract}
Resumo
Atualmente, o crescente aumento populacional tem tornado, tanto a disponibilidade quanto a qualidade da água, um assunto preocupante, devido a isto as águas consideradas de qualidade inferior, tais como as águas residuárias, têm como alternativa a reutilização. Se combinada à piscicultura, possibilita a produção de pescado, a partir de águas que não teriam uso convencional. Desta maneira, neste estudo foram realizadas análises físico-químicas e microbiológicas em dois tanques de estabilização em série localizados na Universidade Federal do Pampa (UNIPAMPA), Campus Uruguaiana, a fim de verificar a possibilidade de reúso destas águas em atividades de piscicultura, considerando os principais parâmetros utilizados para o cultivo em comparação com o estabelecido na resolução 357/05 do CONAMA. No período entre abril/2012 e março/2013 as amostras foram coletadas em dois tanques de estabilização: primário (T1) e secundário (T2). As análises físico-químicas foram realizadas mensalmente pelos métodos colorimétrico e de Winkler, como também em aparelhagens específicas (pHmetro, turbidímetro, multiparâmetro e disco de Secchi). Enquanto os parâmetros microbiológicos foram realizados bimestralmente pela técnica de fermentação em tubos múltiplos (número mais provável). Os resultados obtidos estiveram de acordo com os limites da resolução em ambos os tanques, bem como com o recomendável na atividade de piscicultura descrito por vários autores. Exceto que, ambos os tanques apresentaram níveis de oxigênio abaixo dos valores contidos na resolução, além de que no T1 o limite preconizado para coliformes termotolerantes foi ultrapassado. Dessa maneira, as análises indicaram que as águas do T2 possuem maior potencial para a piscicultura.

Palavras-chave: Águas residuárias, Análises físico-químicas, Análises microbiológicas, Piscicultura, Reúso de água.
\end{abstract}

\begin{abstract}
Currently, the increasing population has been becoming, both the availability and quality of water, a matter of concern, due to this the waters considered inferior, such as wastewater, it has alternative reuse. If combined with fish farming, enables the production of fish from water which would not have the conventional use. Thus, in this study was performed physico-chemical and microbiological analyses in two serial stabilization ponds located at Federal University of Pampa (UNIPAMPA), Campus Uruguaiana in order to verify the possibility of reuse of these waters for fish farming activities, considering the main parameters used for fish farming compared with the provisions of CONAMA Resolution no. $357,2005$. In the period between April 2012 and March 2013, water samples were collected in two stabilization ponds: the primary pond (T1) and secondary pond (T2). The physico-chemical analyses were performed monthly by the methods: colorimetric and Winkler, as well as with specific apparatus ( $p H$ meter, turbidity meter, multiparameter meter and Secchi disk). While the microbiological analyses were performed every two months by the multiple-tube fermentation technique (most probable number). The results obtained were in according to the limits of resolution at both ponds, as well as with the recommended parameters used at fish farming described by several authors. Except which both ponds showed oxygen levels below the values contained in the resolution, besides which the T1 exceeds the recommended limit for fecal coliform. Thus, the analyses indicated which T2 waters have the better potential for fish farming.

Keywords: Residuary waters, Physico-chemical analyzes, Microbiological analyzes, Fish farming, Water reuse.
\end{abstract}




\section{Introdução}

Nas últimas décadas, o conjunto das atividades humanas concomitante com o crescente aumento demográfico tem estimulado a conscientização quanto à importância dos recursos naturais, principalmente os hídricos, que englobando questões tanto sobre sua qualidade quanto sobre seu uso racional, visando controlar perdas e desperdícios. A política de gestão deste recurso já tem algumas décadas. Em 1958, o Conselho Econômico e Social das Nações Unidas, um dos órgãos mais importantes da ONU, estabeleceu uma política de gestão para áreas carentes de recursos hídricos, na qual nenhuma água de boa qualidade, exceto que haja grande disponibilidade, deve ser utilizada para atividades que toleram o uso das águas de qualidade inferior (HESPANHOL, 2003).

Atualmente, com a conscientização ambiental, esta política tem sido empregada em diversas áreas visando um reaproveitamento deste recurso considerado de qualidade inferior em atividades produtivas, as quais demandariam um volume considerável de água, que poderia abastecer comunidades.

Uma destas águas consideradas de qualidade inferior são as águas residuárias, as quais têm origem doméstica (incluindo residências, instituições e comércio), águas de infiltração e/ou de despejos industriais, as quais são de diversas origens e de tipos de indústrias (VON SPERLING, 2005).

Segundo Bartone (1986), não há outro processo convencional de tratamento de águas residuárias, exceto a desinfecção, que pode ser igualado à capacidade das lagoas de estabilização para eliminar agentes patogênicos. Devido a este tratamento ser biológico, possibilita uma opção tecnológica apropriada para os países tropicais em desenvolvimento e se associada à reutilização pode servir como uma medida eficaz de controle sanitário.

O uso das águas residuárias domésticas em tanques de peixes é tão antigo quanto à própria piscicultura. Porém, não é possível utilizar a mesma infraestrutura do sistema de tratamento concomitante com a piscicultura, pois deve-se utilizar as águas tratadas em tanques construídos especificamente para a atividade de cultivo (MOSCOSO; GALECIO, 1978).

Esta combinação é essencial para a geração de produtos, a partir da reutilização de recursos hídricos considerados de qualidade inferior e, consequentemente, impedir que estas venham a contaminar cursos d'água, constituindo uma alternativa ecologicamente correta, a qual aperfeiçoará o uso desse recurso tão vital. Devido ao produto desta atividade ter como principal finalidade o consumo humano, então é de suma importância que esteja nos padrões estabelecidos na legislação vigente para assegurar a qualidade do produto e, com isso, a proteção da saúde pública.

Portanto, este estudo visou analisar os parâ- metros físico-químicos e microbiológicos de águas residuárias de dois tanques de estabilização em série situados na fronteira oeste do estado do Rio Grande do Sul e averiguar a possibilidade de reúso destas águas em atividades de piscicultura, considerando os principais parâmetros estabelecidos pela resolução 357/05 do Conselho Nacional do Meio Ambiente (CONAMA), para utilização de águas com fins na Aquicultura-Classe 2, a qual engloba a piscicultura.

\section{Material e Método}

\subsection{Descrição do local}

O estudo foi realizado no período entre abril de 2012 e março de 2013, em dois tanques de estabilização escavados e sem revestimento, em série: tanque primário (T1), o qual é receptor de águas residuárias provenientes originalmente de um poço tubular e que, posteriormente, abastece os laboratórios; e tanque secundário (T2). $\mathrm{O}$ despejo do efluente em T1 ocorre através de dois canos de PVC (C1 e C2). Este efluente deságua no T2 por meio de um canal de ligação e, após, são despejadas no local em direção ao córrego Felizardo. Os tanques estão localizados na latitude de $29^{\circ} 50^{\prime} 20^{\prime \prime} \mathrm{S} ; 29^{\circ} 50^{\prime} 52^{\prime \prime} \mathrm{S}$, e longitude de $57^{\circ} 6^{\prime} 23^{\prime \prime} \mathrm{O} ; 57^{\circ} 6^{\prime} 14^{\prime \prime} \mathrm{O}$, respectivamente para T1 e T2, conforme podem ser observados na Figura 1 , na UNIPAMPA.

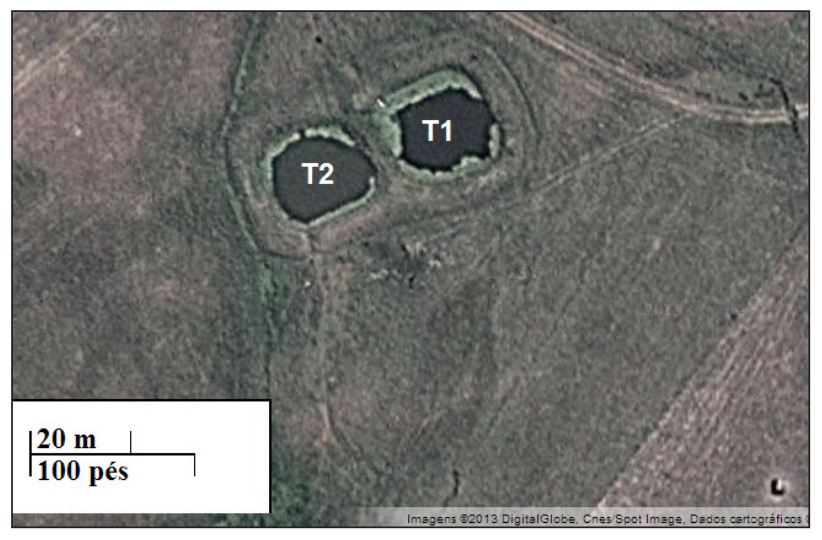

Figura 1: Vista aérea dos tanques de estabilização em série, localizados na UNIPAMPA, Campus Uruguaiana visualizados a partir da imagem Google Earth

Fonte: www.google.com.br/intl/pt-BR/earth/.../ge/agree.html (adaptado).

Os tanques foram medidos com um GPS Garmin Legend H e, a partir do programa Track Maker Professional (Satellite Navigation Program), foi determinada a área total dos tanques em $533 \mathrm{~m}^{2}$ e $537 \mathrm{~m}^{2}$, respectivamente para T1 e T2. A vazão de abastecimento do T1 foi medida semanalmente durante os meses de abril e maio, e então foi calculada a vazão média de abastecimento do $\mathrm{C} 1$ igual a 
$151 \mathrm{~L} / \mathrm{h}$ e no C2 correspondeu a $96 \mathrm{~L} / \mathrm{h}$, ambos com fluxo descontínuo.

\subsection{Coleta das amostras}

A metodologia utilizada para esterilização do material, amostragem, acondicionamento e transporte seguiu as recomendações de acordo com APHA et al. (1992).

As amostras de ambos os tanques foram coletadas na superfície da água às 9 h e 45 min, sendo que as análises dos parâmetros físico-químicos foram realizadas mensalmente in loco, exceto as análises do oxigênio dissolvido (OD), demanda bioquímica de oxigênio $\left(\mathrm{DBO}_{5}\right)$, turbidez e $\mathrm{pH}$.

Já para as análises microbiológicas, as amostras foram coletadas bimestralmente, conforme estabelecido na resolução 357/05 do CONAMA, em balões de Erlenmeyer, vedados com buchas (gaze e algodão) e previamente autoclavados.

\subsection{Parâmetros físico-químicos}

As análises dos parâmetros físico-químicos realizadas foram temperatura do ar e da água $\left({ }^{\circ} \mathrm{C}\right)$, salinidade e condutividade elétrica (C.E. medida em $\mathrm{mS} / \mathrm{cm}$ ), medidos com o multiparâmetro Hanna HI9828. Além disso, através do Alfakit Kit Produtor de Água Doce, foram quantificadas a transparência $(\mathrm{cm})$, amônia $(\mathrm{mg} / \mathrm{L})$, nitrito $(\mathrm{mg} / \mathrm{L})$, alcalinidade $(\mathrm{mg} / \mathrm{L}$ de $\left.\mathrm{CaCO}_{3}\right)$ e dureza total $\left(\mathrm{mg} / \mathrm{L}\right.$ de $\left.\mathrm{CaCO}_{3}\right)$, realizados pelo método colorimétrico por comparação com o padrão, conforme as instruções do fabricante. Através do turbidímetro Hanna HI98703 foi quantificada a turbidez (UNT); já o $\mathrm{pH}$, por meio do pHmetro Gehaka PG1800. Para a determinação do OD e da $\mathrm{DBO}_{5}$, foi utilizado o método de Strickland; Parsons (1972), baseado no método iodométrico clássico de Winkler descrito em Baumgarten et al. (1996).

Seguindo a mesma metodologia, também foram coletadas e analisadas amostras em dois pontos que são fontes de abastecimento dos tanques de estabilização. Estas coletas foram realizadas durante a manhã e a tarde em ambos os tanques (T1 e T2), no mês de março de 2013. Sendo estes pontos: A1, proveniente da torneira do Núcleo de Pesquisas Ictiológicas,
Limnológicas e Aquicultura da Bacia do Rio Uruguai (NUPILABRU), e A2, correspondente à torneira da caixa d'água. Ambas foram consideradas como as águas de origem do efluente.

\subsection{Análises microbiológicas}

As amostras foram analisadas pela técnica de fermentação em tubos múltiplos (número mais provável - NMP) de acordo com APHA et al. (1992), da qual foram utilizadas 3 repetições de tubos em 3 diluições de água diferentes.

Tanto os coliformes totais (CT) quanto os termotolerantes (CF) foram analisados nas diluições $10^{-3}, 10^{-4}$ e $10^{-5}$, pois acreditava-se que a concentração dos mesmos seria muito alta devido a estas águas serem residuárias. Não se considerou inicialmente que, ao aumentar a diluição, consequentemente elevava-se o NMP, o qual ficava impreciso garantir se o resultado era maior ou menor que o nível requerido na resolução. Em vista disto, a partir da segunda amostragem (junho) as diluições foram alteradas para $10^{-1}, 10^{-2}$ e $10^{-3}$.

\subsection{Coleta de peixes}

Durante o mês de março de 2013, foram colocados covos em ambos os tanques a fim de averiguar a presença de peixes nos mesmos, como indicadores biológicos da qualidade da água.

\section{Resultados}

\subsection{Análises físico-químicas}

A faixa de variação da temperatura do ar de ambos os tanques está disposta na Tabela 1, conforme as estações do ano.

A temperatura da água em T1 e T2 está disposta na Tabela 2, de acordo com a estação e em comparação com o estudo realizado por Garcia et al. (2008).

A faixa de variação na concentração de OD foi de $3,50 \pm 2,55 \mathrm{mg} / \mathrm{L}$ no T1 e 3,16 $\pm 2,23 \mathrm{mg} / \mathrm{L}$ no T2, já a faixa de variação da $\mathrm{DBO}_{5}$ correspondeu a 3,24 $\pm 2,43$ $\mathrm{mg} / \mathrm{L}$ no T1 e 2,39 $\pm 1,54 \mathrm{mg} / \mathrm{L}$ no T2. Os dados de ambos os tanques podem ser observados na Figura 2, a e b.

Tabela 1 - Temperaturas médias do ar em ambos os tanques.

\begin{tabular}{ccccc}
\hline \multirow{2}{*}{ Estação } & \multicolumn{2}{c}{ T1 } & \multicolumn{2}{c}{ T2 } \\
\cline { 2 - 5 } & Média & Desv. Pad. & Média & Desv. Pad. \\
\hline Outono & 17,43 & 4,67 & 17,34 & 4,74 \\
Inverno & 17,54 & 5,72 & 15,75 & 6,55 \\
Primavera & 25,84 & 6,28 & 26,00 & 6,36 \\
Verão & 24,54 & 1,76 & 24,56 & 1,75 \\
\hline
\end{tabular}


Tabela 2 - Comparativo das temperaturas da água em ambos os tanques com um estudo.

\begin{tabular}{ccccccc}
\hline \multirow{2}{*}{ Estação } & \multicolumn{2}{c}{ T1 } & \multicolumn{2}{c}{ T2 } & \multicolumn{2}{c}{ Garcia et al.(2008) } \\
\cline { 2 - 7 } & Média & Desv. Pad. & Média & Desv. Pad. & Min. & Máx. \\
\hline Outono & 16,97 & 3,63 & 17,47 & 3,71 & 17 & 25 \\
Inverno & 15,78 & 6,05 & 15,81 & 5,64 & 9 & 17 \\
Primavera & 24,13 & 3,21 & 24,50 & 3,31 & 14 & 21 \\
Verão & 22,16 & 1,11 & 22,79 & 1,11 & 16 & 28 \\
\hline
\end{tabular}

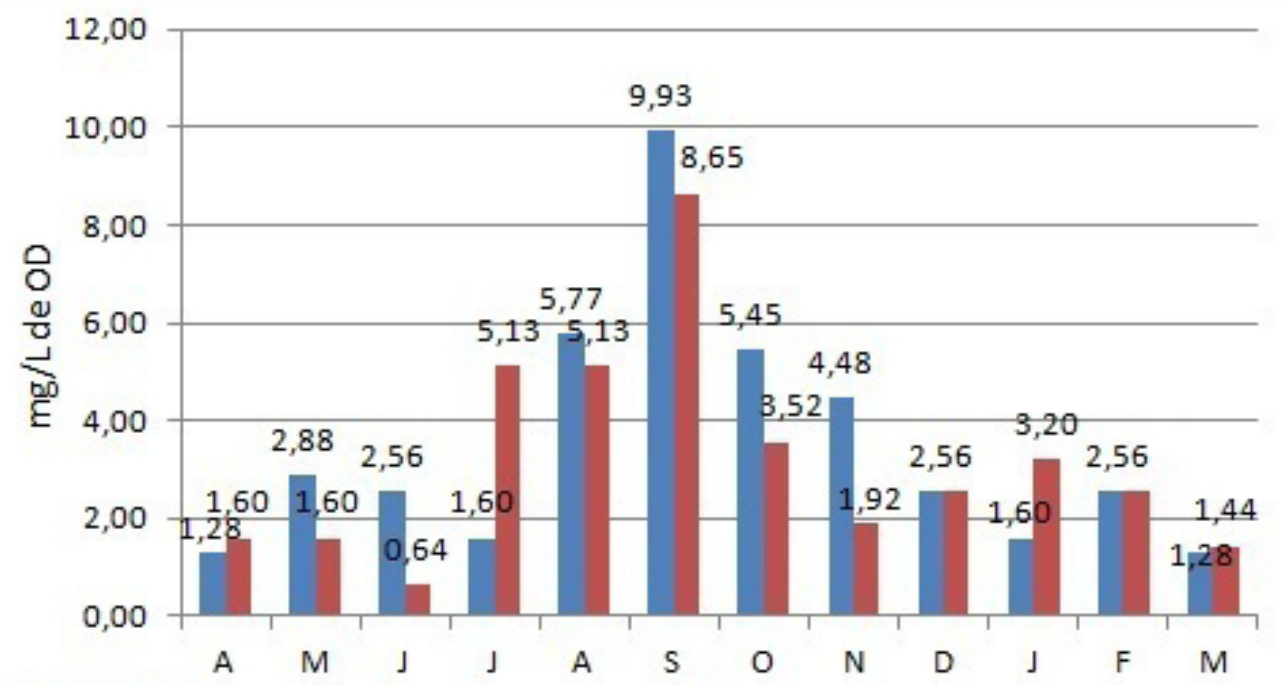

T1 T2

Mês

(a)

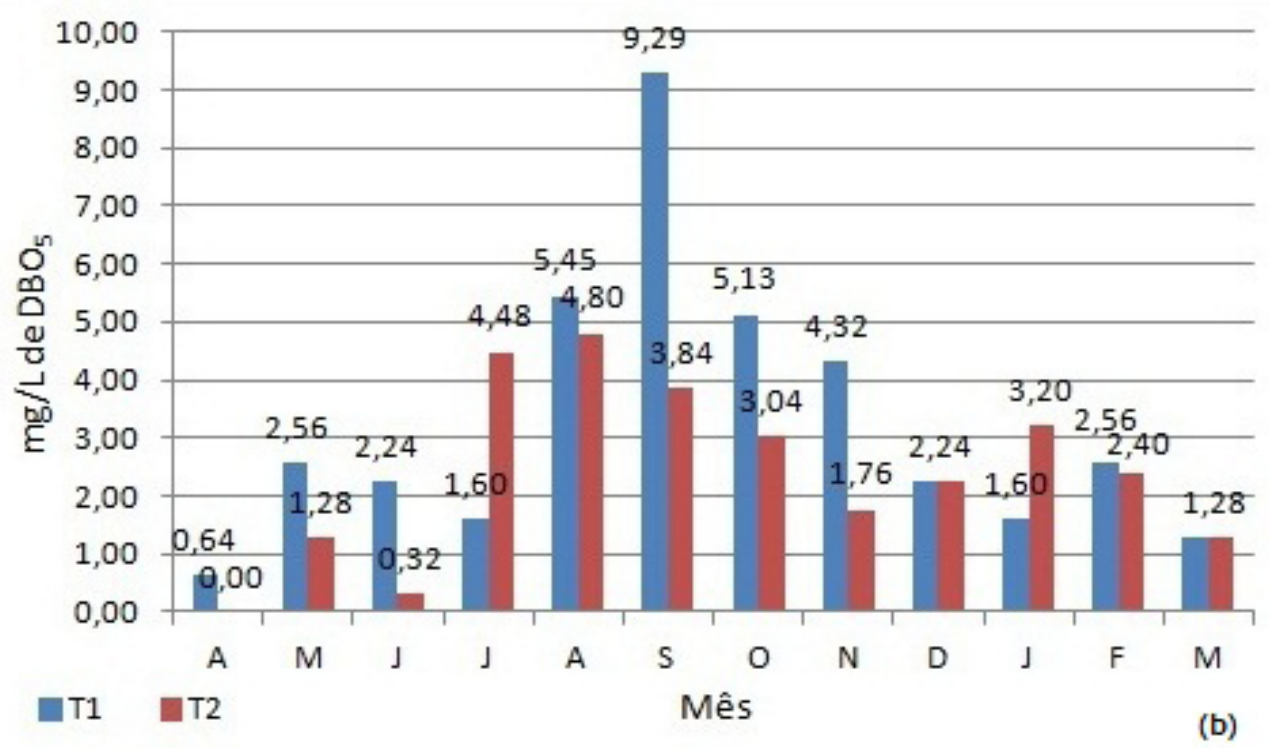

Figura 2: Concentração de $\mathrm{OD}(\mathrm{a})$ e $\mathrm{DBO}_{5}$ (b) em mg/L dos tanques de estabilização localizados na UNIPAMPA (Obs. M=Maio). 


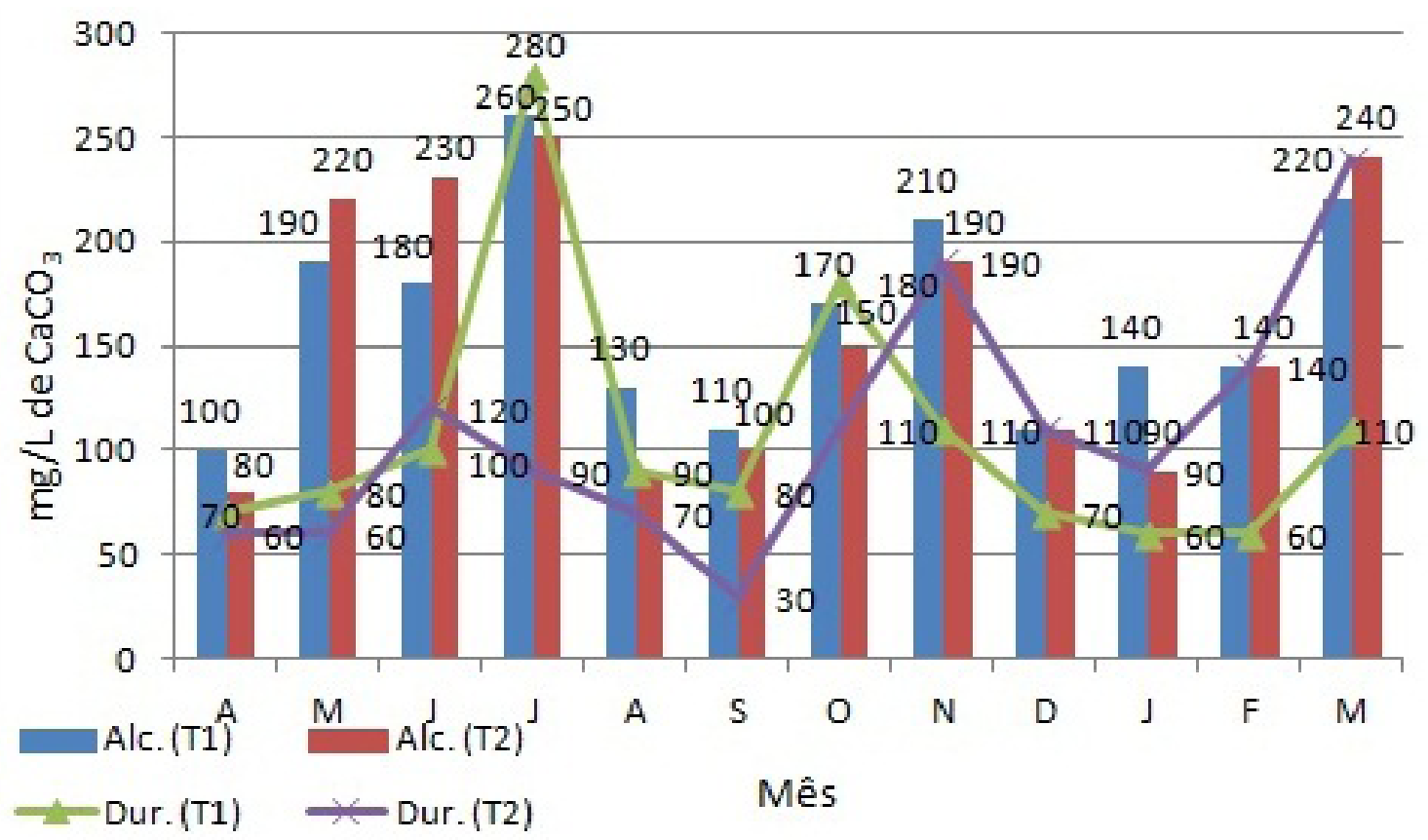

Figura 3: Alcalinidade e dureza total em mg/L de $\mathrm{CaCO}_{3}$ dos tanques de estabilização situados na UNIPAMPA.

A faixa de variação da alcalinidade total no $\mathrm{T} 1$ foi de $163,33 \pm 50,15 \mathrm{mg} / \mathrm{L}$ de $\mathrm{CaCO}_{3}$ e a faixa de variação da dureza total igual a 107,50 $\pm 63,41 \mathrm{mg} / \mathrm{L}$ de $\mathrm{CaCO}_{3}$. Já no T2, a alcalinidade total correspondeu a $157,50 \pm 65,10 \mathrm{mg} / \mathrm{L}$ de $\mathrm{CaCO}_{3}$ e a dureza total variou de $76,67 \pm 27,74 \mathrm{mg} / \mathrm{L}$ de $\mathrm{CaCO}_{3}$. Os dados referentes a ambos os tanques estão dispostos na Figura 3, já a relação dureza total: alcalinidade total nestes correspondeu a 0,66 e 0,49 , respectivamente em T1 e T2.

Enquanto, conforme pode ser observado na
Figura 4, a faixa de variação do $\mathrm{pH}$ foi igual a 7,81 $\pm 0,73$

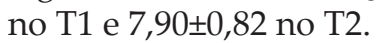

A faixa de variação das concentrações de amônia e nitrito foram de 0,06 $\pm 0,08 \mathrm{mg} / \mathrm{L}$ e 0,20 $\pm 0,35$ $\mathrm{mg} / \mathrm{L}$, respectivamente no T1. Já a concentração de amônia, no T2, foi de $0,08 \pm 0,10 \mathrm{mg} / \mathrm{L}$ e de nitrito correspondeu a $0,25 \pm 0,44 \mathrm{mg} / \mathrm{L}$. Ambos os parâmetros estão dispostos na Figura 5, a e b.

No T1, a transparência média foi igual a $14,25 \pm 8,75 \mathrm{~cm}$ e a turbidez média correspondeu de

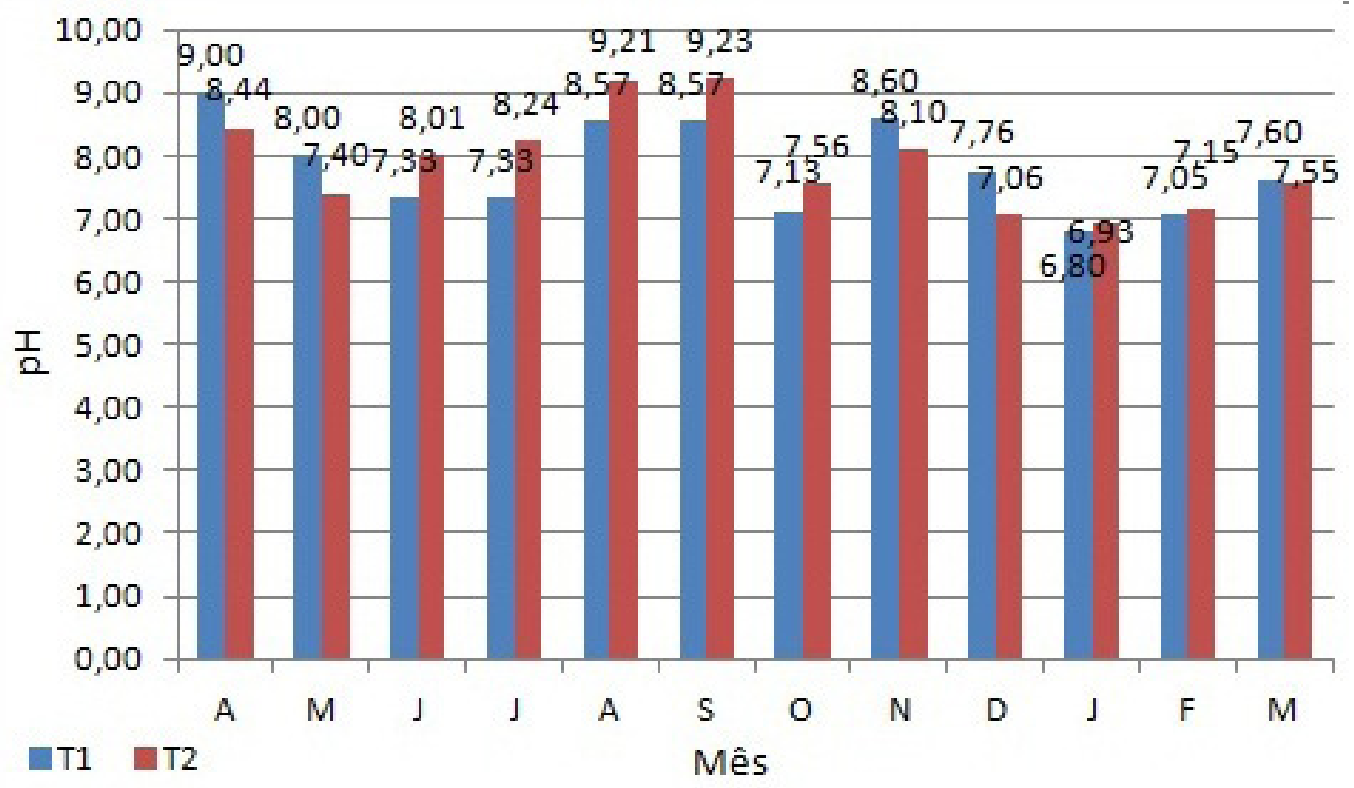

Figura 4: Potencial hidrogeniônico $(\mathrm{pH})$ dos tanques de estabilização localizados na UNIPAMPA. 

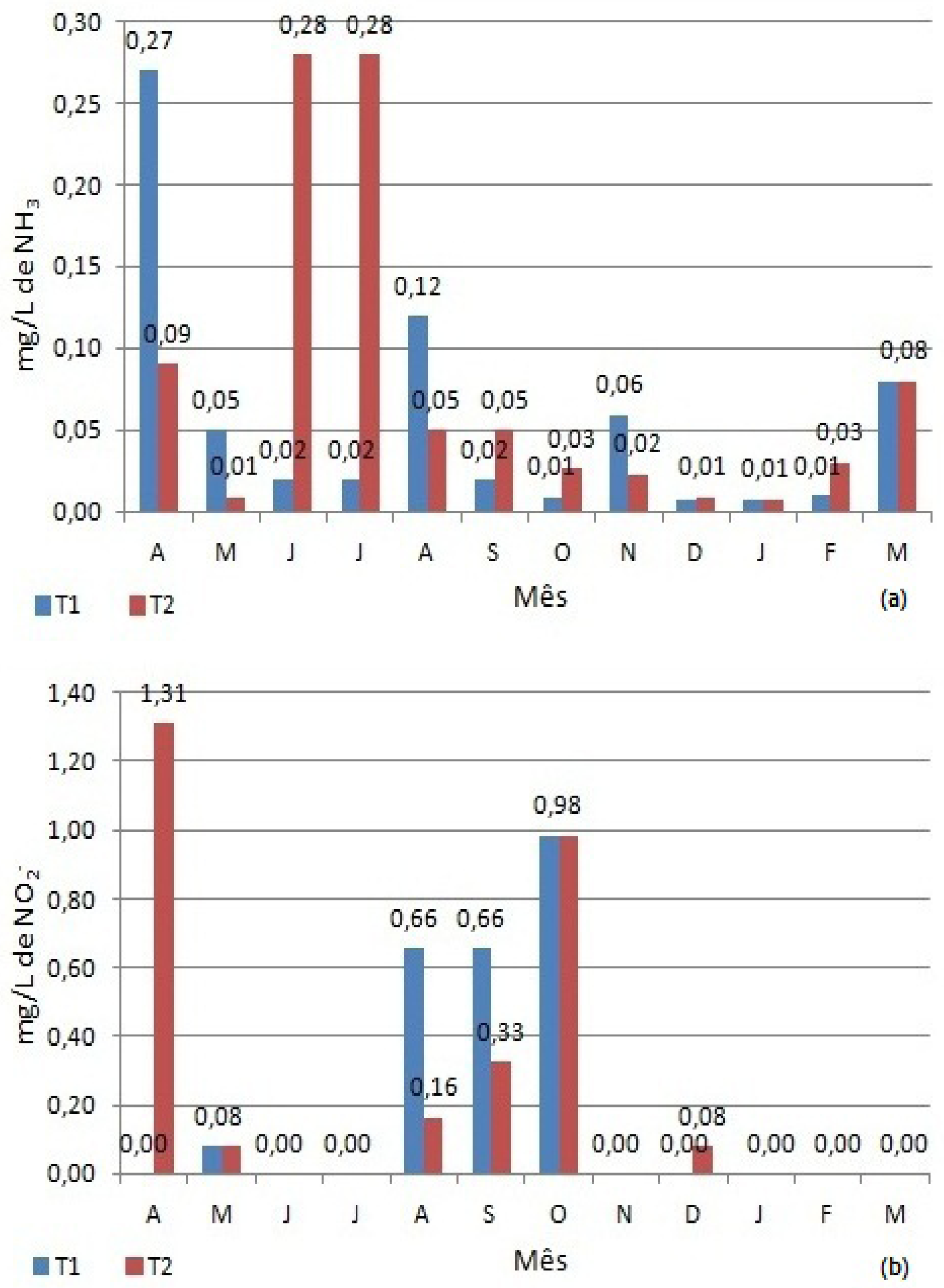

Figura 5: Concentração de amônia (a) e nitrito (b) em mg/L dos tanques de estabilização situados na UNIPAMPA.

$52,88 \pm 41,27$ UNT, enquanto, no T2, foi igual a $13,04 \pm 6,82$ cm e 51,62 $\pm 33,53$ UNT, respectivamente. A faixa de variação de ambos os parâmetros em T1 e T2 estão dispostos na Figura 6, a e b.

No T1 a C.E. e a salinidade foi de $0,31 \pm 0,08$ $\mathrm{mS} / \mathrm{cm}$ e $0,15 \pm 0,04$, respectivamente, já no T2 a C.E. 


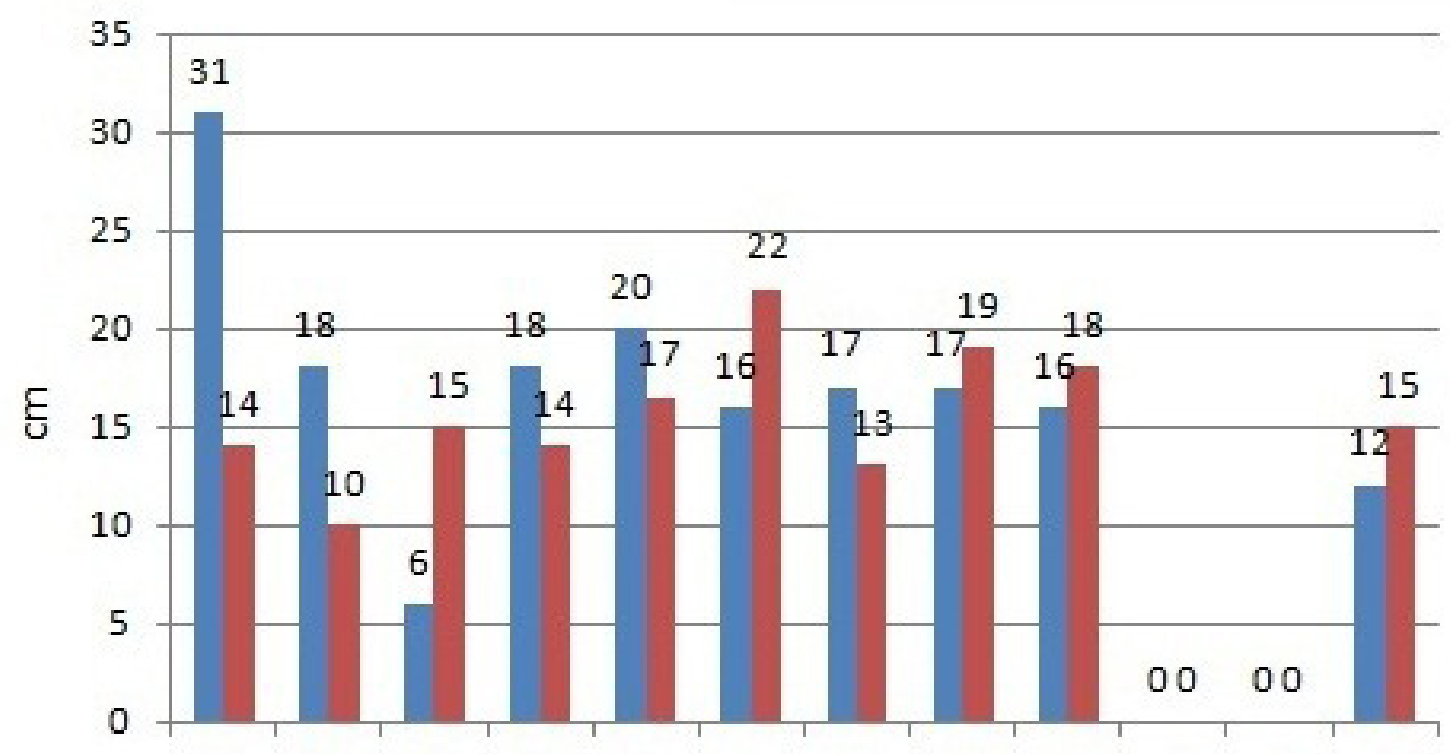

\section{- 1 1}
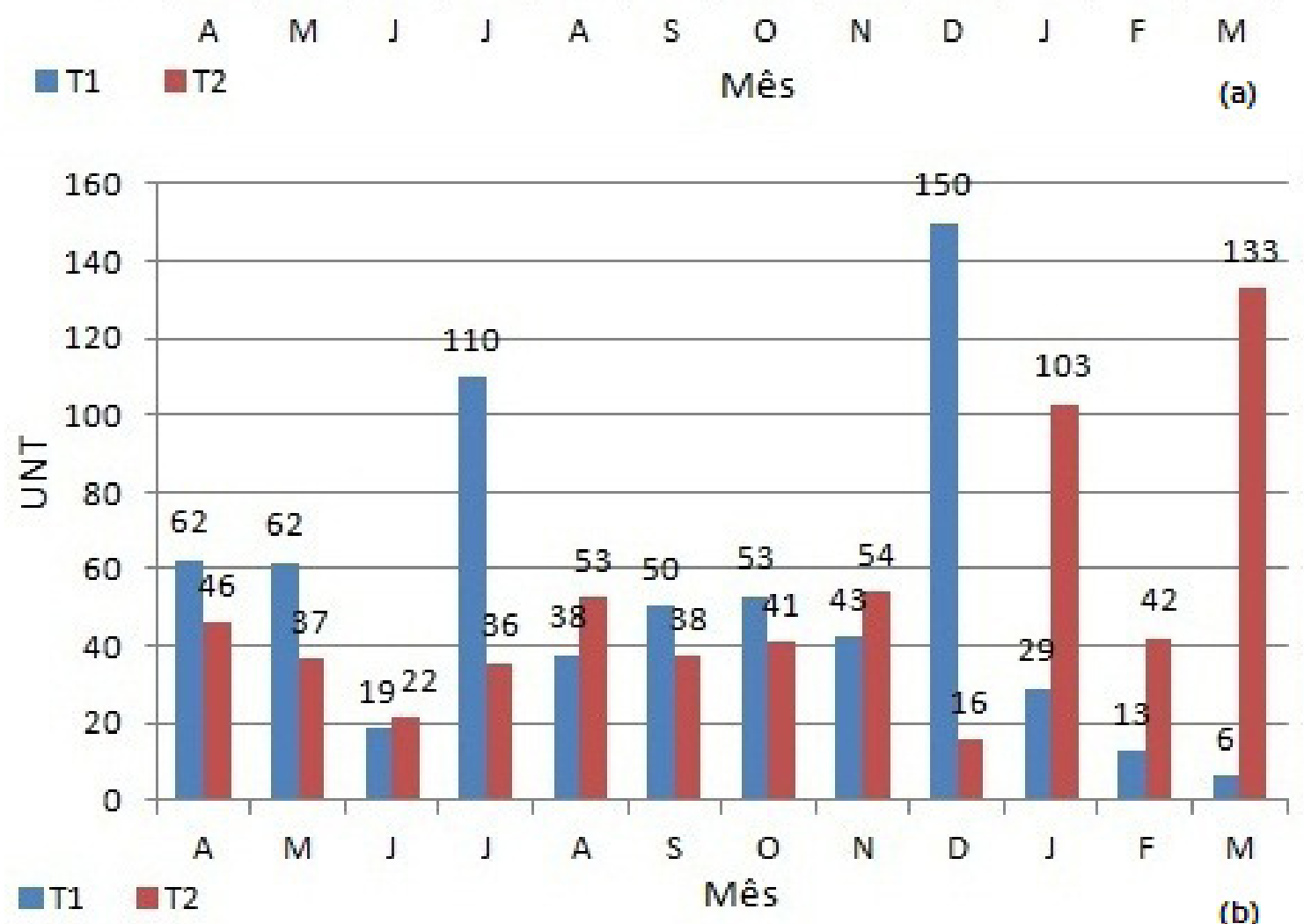

Figura 6: Transparência (a) em cm e turbidez (b) em UNT dos tanques de estabilização localizados na UNIPAMPA.

foi de $0,29 \pm 0,10 \mathrm{mS} / \mathrm{cm}$ e a salinidade correspondeu a $0,14 \pm 0,05$. Na Figura 7 , está disposta a faixa de variação dos dados referentes a ambos os parâmetros.
As análises dos parâmetros físico-químicos das fontes de abastecimento, bem como dos tanques de estabilização, podem ser observadas na Tabela 3. 


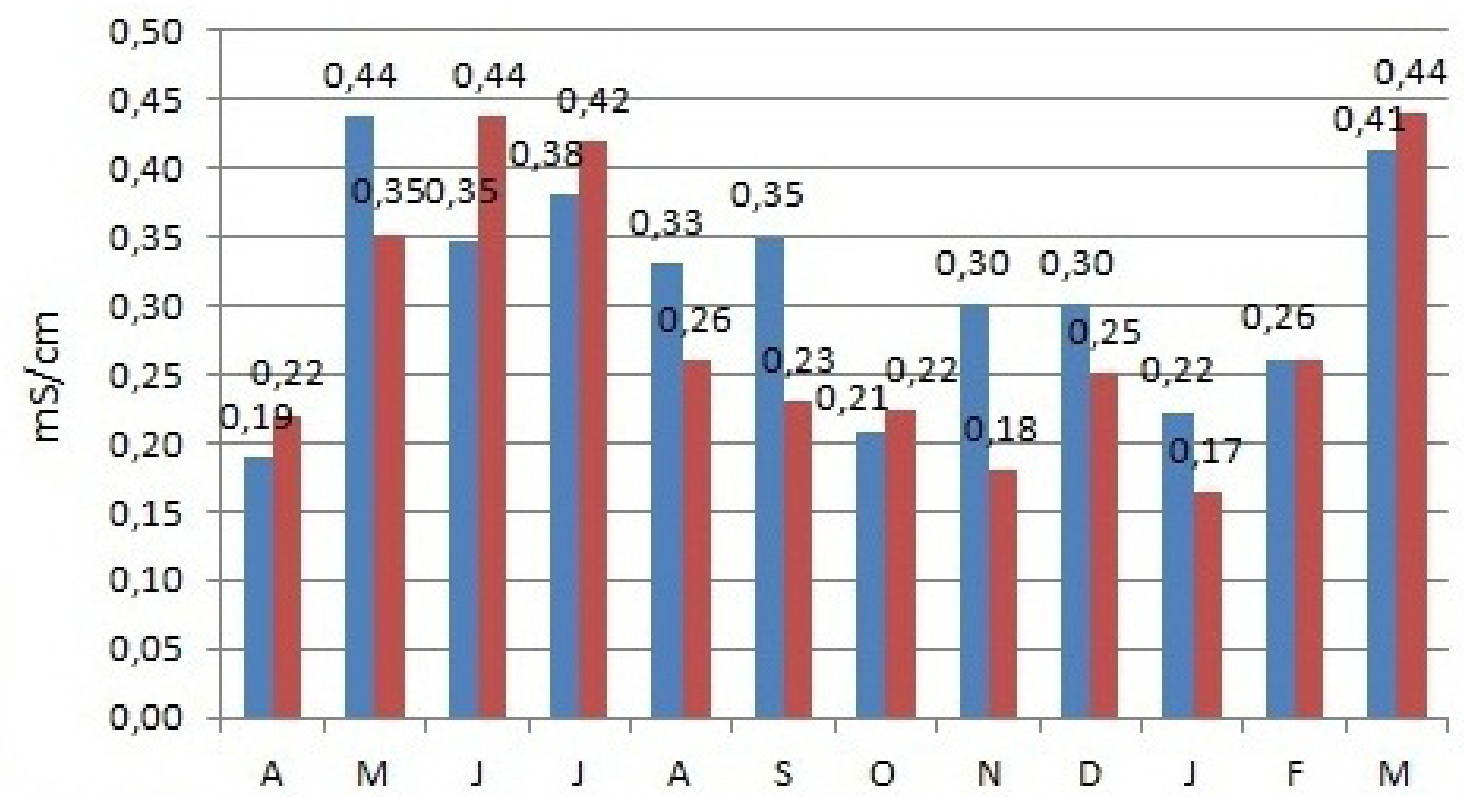

T1 12

Mês

(a)

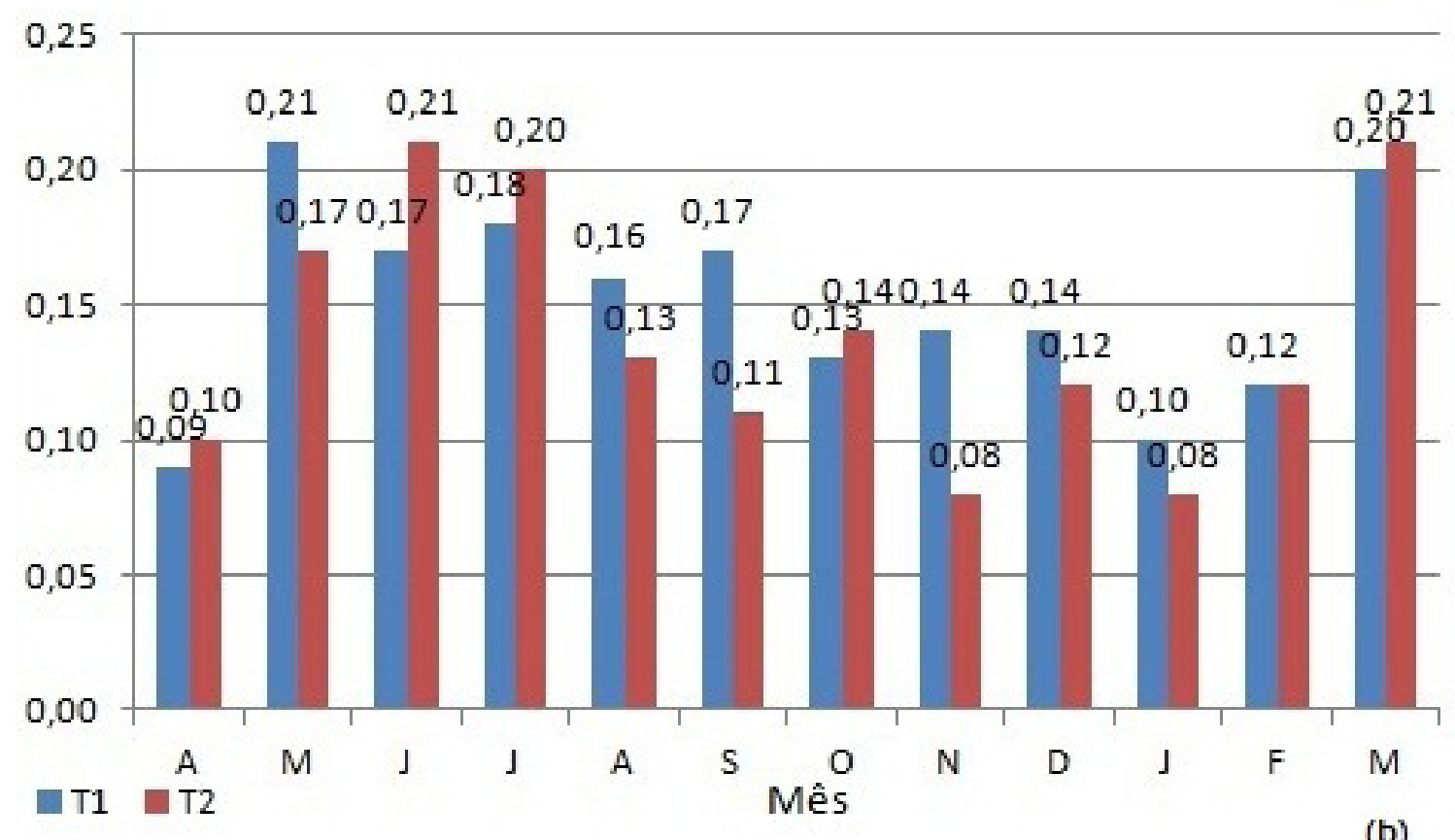

(b)

Figura 7: Condutividade elétrica (mS/cm) (a) e a salinidade (b) dos tanques de estabilização situados na UNIPAMPA.

\subsection{Análises microbiológicas}

Os valores do NMP de CT e CF encontrados nas amostras analisadas podem ser observados na
Tabela 4 e 5, sendo que os CT, no T1, ficaram entre 360 a $<30.000 \mathrm{NMP} / 100 \mathrm{~mL}$ enquanto, no T2, na faixa de 730 a $<30.000$ NMP/100 mL. Já os CF em T1 cor- 
Tabela 3 - Análise das águas de origem dos tanques de estabilização. Torneira do NUPILABRU (A1) e torneira da caixa d'água (A2).

\begin{tabular}{ccccccccc}
\hline \multirow{2}{*}{ Parâmetro } & \multicolumn{2}{c}{ T1 } & \multicolumn{2}{c}{ T2 } & \multicolumn{2}{c}{ A1 } & \multicolumn{2}{c}{ A2 } \\
\cline { 2 - 9 } & $\mathbf{M}$ & $\mathbf{T}$ & $\mathbf{M}$ & $\mathbf{T}$ & $\mathbf{M}$ & $\mathbf{T}$ & $\mathbf{M}$ & $\mathbf{T}$ \\
\hline Temp. Ar & 23,74 & 25,33 & 23,80 & 25,40 & - & - & - & - \\
Temp. Água & 21,62 & 26,33 & 21,54 & 23,74 & 24,18 & 26,01 & 24,22 & 24,66 \\
\hline OD & 1,28 & 2,66 & 1,44 & 4,53 & 5,05 & 4,43 & 3,50 & 2,00 \\
Alcalinidade & 220 & 220 & 240 & 240 & 310 & 300 & 310 & 300 \\
\hline Dureza & 110 & 120 & 110 & 100 & 100 & 100 & 100 & 100 \\
PH & 7,60 & 7,60 & 7,55 & 7,69 & 7,98 & 8,24 & 7,95 & 8,05 \\
Amônia & 0,08 & 0,08 & 0,08 & 0,08 & 0,08 & 0,28 & 0,08 & 0,28 \\
Nitrito & 0 & 0 & 0 & 0 & 0 & 0 & 0 & 0 \\
C.E. & 0,41 & 0,45 & 0,44 & 0,46 & 0,53 & 0,54 & 0,54 & 0,54 \\
\hline Salinidade & 0,20 & 0,22 & 0,21 & 0,22 & 0,26 & 0,26 & 0,26 & 0,26 \\
\hline
\end{tabular}

responderam à faixa de $<300 \mathrm{a}<30.000 \mathrm{NMP} / 100 \mathrm{~mL}$, sendo que $66,67 \%$ das amostras estavam de acordo com a resolução, enquanto a faixa do T2 foi entre $<300$ a $<30.000 \mathrm{NMP} / 100 \mathrm{~mL}$, e $83,33 \%$ das amostras estavam de acordo com a resolução.

\subsection{Coleta de peixes}

No T1, foram identificados alevinos de traíra (Hoplias malabaricus), lambari spp., barrigudinho (Phalloceros sp.) e mussum (Synbranchus marmoratus). Enquanto, no T2, foram observados vários espécimes de carpas (família Cyprinidae), bem como um exemplar adulto de traíra e lambaris, além de diversos moluscos, insetos e macrófitas aquáticas.

\section{Discussão}

\subsection{Análises físico-químicas}

Os dados coletados referentes à temperatura do ar sugerem que houve pequenas variações entre os tanques, porém a maioria apresentou o desvio padrão alto, indicando grau de variabilidade nas variáveis.

De acordo com o estudo realizado por Garcia

Tabela 4 - Coliformes totais e termotolerantes (NMP/100 mL) presentes no T1.

\begin{tabular}{ccccccc}
\hline \multirow{2}{*}{ Parâmetro } & \multicolumn{5}{c}{ Mês } \\
\cline { 2 - 7 } & $\mathbf{M}$ & $\mathbf{J}$ & $\mathbf{S}$ & $\mathbf{N}$ & $\mathrm{J}$ & $\mathbf{M}$ \\
\hline $\mathbf{n}$ CT & $<3.000$ & 2.300 & 2.300 & 360 & 4.300 & 730 \\
$\mathrm{CF}$ & $<3.000$ & 2.300 & 910 & $<300$ & 360 & $<300$ \\
\hline
\end{tabular}

Tabela 5 - Coliformes totais e termotolerantes (NMP/100 mL) presentes no T2.

\begin{tabular}{ccccccc}
\hline \multirow{2}{*}{ Parâmetro } & \multicolumn{5}{c}{ Mês } \\
\cline { 2 - 7 } & $\mathbf{M}$ & $\mathbf{J}$ & $\mathbf{S}$ & $\mathbf{N}$ & $\mathrm{J}$ & $\mathbf{M}$ \\
\hline \multirow{2}{*}{ CT } & $<3.000$ & 1.900 & 730 & 910 & 910 & 2.300 \\
CF & $<3.000$ & 620 & 300 & 360 & 360 & $<300$ \\
\hline
\end{tabular}


et al. (2008), nas cidades do estado do Rio Grande do Sul, incluindo Uruguaiana, durante os anos de 1996 a 2004 , as temperaturas da água oscilaram entre 16 a $28^{\circ} \mathrm{C}$ no verão; 17 a $25^{\circ} \mathrm{C}$ no outono; 14 a $17^{\circ} \mathrm{C}$ no inverno (chegando a $9^{\circ} \mathrm{C}$ nos meses mais frios); e na faixa de 14 e $21^{\circ} \mathrm{C}$ na primavera. Os dados registrados pelos autores referidos foram semelhantes ao encontrado no presente estudo, conforme pode ser observado na Tabela 2. O mês de julho foi o mais frio do ano, quando as temperaturas estiveram próximas a $9^{\circ} \mathrm{C}$ em T1 e T2, tanto as temperaturas do ar quanto da água foram influenciadas pelas condições climáticas.

Ainda neste mesmo referencial teórico, foram citadas espécies nativas resistentes às baixas temperaturas do inverno, que são o jundiá (Rhamdia quelen), traíra, pintado (Pimelodus maculatus), bem como as espécies exóticas (carpas). Destas espécies citadas, foram observados, durante os meses de inverno, vários espécimes de traíras e carpas no T2.

$\mathrm{Na}$ maioria dos meses, os níveis de OD, em ambos os tanques, estiveram abaixo do mínimo estabelecido na resolução (5 mg/L de OD), conforme pode ser observado na Figura 2 (a). Entretanto, diversas espécies de peixes toleram por períodos prolongados a faixa entre 2 a $3 \mathrm{mg} / \mathrm{L}$ de OD (KUBITZA, 1998b). Este fato é evidenciado pela presença de alevinos de traíra, lambari, barrigudinho e mussum no T1, enquanto no T2 foram encontradas carpas, traíras e lambaris. Baldisserotto (2009) afirmou que a traíra e a carpa comum são facilmente adaptáveis em águas com baixa pressão de oxigênio. Em Felizatto (2000), é mencionado que a carpa prateada sobrevive em extremas condições de falta de oxigênio (entre 0,3 a 1,1 mg/L). Enquanto em Montenegro (2009), é referido que o mussum vive em águas com pouco oxigênio e durante as secas sobrevivem enterrados na lama, neste caso, no lodo.

Esses níveis de oxigênio baixos devem-se à baixa renovação das águas, alta quantidade de matéria orgânica, bem como ao excesso de macrófitas. Em Tavares (2008) é citado que as lemnáceas são empregadas em sistemas de tratamento secundário visando a recuperação de nutrientes, porém estas formam uma manta superficial na água, a qual inibe a entrada de oxigênio nela, tanto por difusão quanto pela produção realizada pelo fitoplâncton.

Já para a $\mathrm{DBO}_{5}$, é estabelecido na resolução, o máximo de $5 \mathrm{mg} / \mathrm{L}$. Ambos os tanques estão de acordo com este limite da resolução, conforme pode ser observado na Figura 2 (b), exceto no T1, durante os meses de agosto, setembro e outubro. O mês de setembro foi o valor mais expressivo devido a um despejo orgânico maior que o habitual associado à presença de micro-organismos (maior que no T2), o qual proporcionou este aumento na $\mathrm{DBO}_{5}$. As pesquisas que abordam o reúso de águas residuárias provenientes de lagoas de estabilização em piscicultura têm a
$\mathrm{DBO}_{5}$ na faixa de uma ou mais unidades logarítmicas acima do presente estudo, devido provavelmente a maior quantidade de matéria orgânica.

A alcalinidade total de ambos os tanques esteve acima de $30 \mathrm{mg} / \mathrm{L}$ de $\mathrm{CaCO}_{3}$ e não ultrapassou $300 \mathrm{mg} / \mathrm{L}$ de $\mathrm{CaCO}_{3}$, conforme pode ser observado na Figura 3. Nesta faixa, haverá bom desenvolvimento no cultivo, sendo uma boa indicação sobre a qualidade de tais sais, pois ajudam na formação de plâncton (KUBITZA, 2000). Conforme citado em Moscoso; Galecio (1978), as altas concentrações de alcalinidade e elevado $\mathrm{pH}$ são características das águas residuárias que estão associadas à piscicultura. Entretanto, esta alcalinidade elevada não se deve somente as águas serem residuárias, pois também tem relação com a água de origem, a qual apresenta alta alcalinidade, conforme pode ser observado na Tabela 3.

Tanto a alcalinidade quanto a dureza em ambos os tanques estiveram acima de $30 \mathrm{mg} / \mathrm{L}$ de $\mathrm{CaCO}_{3}$ (Figura 3), isto garante bom funcionamento do sistema tampão da água (KUBITZA, 1998a). A relação da dureza e alcalinidade indica, em ambos os tanques, que há alta alcalinidade e baixa dureza. Neste caso, de acordo com Baldisserotto (2009), os carbonatos e bicarbonatos estão associados ao $\mathrm{K}^{+} \mathrm{e}$ $\mathrm{Na}^{+}$, sendo que esta dureza temporária ou temporal pode ser eliminada por fervura.

Os valores da relação dureza total: alcalinidade total foi abaixo de 1,0 em ambos os tanques, semelhante aos resultados encontrados por Santos (2012). Esses valores baixos na relação dureza total: alcalinidade total podem explicar o valor médio de $\mathrm{pH}$ muito alto nos tanques no horário da manhã, pois é citado neste que para a manutenção do efeito tampão a relação dureza total:alcalinidade total deve ser mantida maior que 1,0 .

Desta forma, pode-se associar o valor elevado do $\mathrm{pH}$ e a ausência de um padrão em ambos os tanques (Figura 4), as características naturais das águas de origem utilizadas em seu abastecimento, dispostas na Tabela 3. Também se deve considerar que, de acordo com Santos (2012), em esgoto tratado, o pH tende a diminuir, devido à presença de ácidos orgânicos que, após dissociados, liberam íons $\mathrm{H}^{+}$na água reduzindo o pH. Entretanto, os tanques apresentaram elevada biomassa algal o que, por meio da fotossíntese, consome essas fontes de carbono e libera íons $\mathrm{OH}^{-}$, tornando- o elevado. Em ambos os tanques, o pH encontrou-se na faixa estabelecida pela resolução, somente o T2, durante os meses de agosto e setembro, ultrapassaram um pouco o limite máximo de 9 , porém a faixa entre 6,5 a 9,5 são consideradas aceitáveis, mesmo sendo acima da faixa ideal (ALBANEZ; MATOS, 2007).

A amônia não ionizada, a qual é tóxica para os peixes, quando for acima de $0,2 \mathrm{mg} / \mathrm{L}$, será suficiente para induzir uma toxidez crônica e, por conseguinte, a diminuição do crescimento e tolerância às doenças 
(KUBITZA, 1998a). No presente estudo, conforme pode ser observado na Figura 5 (a), apenas no mês de abril, em T1, a amônia esteve acima de $0,2 \mathrm{mg} / \mathrm{L}$, provavelmente devido à baixa concentração de $\mathrm{OD}$ não houve a nitrificação (PEREIRA; MERCANTE, 2005). Já no T2, a amônia esteve acima desta faixa durante os meses de junho e julho, sendo o primeiro mês devido à baixa concentração de OD, enquanto no segundo mês o OD ainda não tinha sido assimilado pelo processo de nitrificação, possivelmente, devido a pouca presença de bactérias do gênero Nitrossomonas. Também deve-se considerar que as águas de origem apresentaram concentração de amônia, acima do recomendado durante a tarde. Porém, ambos os tanques apresentaram valor médio de acordo com o recomendável e mesmo durante os meses que ultrapassaram, não foram um fator limitante, pois ambos os tanques continham peixes em diferentes estágios de desenvolvimento.

De acordo com Ostrensky; Boeger (1998), os níveis de nitrito, a partir de $0,5 \mathrm{mg} / \mathrm{L}$, geralmente passam a ser tóxicos para os peixes, acarretando a diminuição das taxas de crescimento e aumento de estresse. Este limite foi ultrapassado, conforme pode ser observado na Figura 5 (b), no T1, durante os meses de agosto, setembro e outubro; enquanto, no T2, correspondeu a abril e outubro. Porém, em um experimento realizado por Lima et al. (2011) com juvenis de jundiá, verificou-se que o nível seguro de nitrito para a fase de crescimento é abaixo de $1,19 \mathrm{mg} / \mathrm{L}$, ou seja, apenas o mês de abril, no T2, estaria acima da faixa segura para o crescimento desta espécie. Na resolução é estabelecido o máximo de $1 \mathrm{mg} / \mathrm{L}$ de nitrito, ambos tanques estão de acordo com a resolução, exceto o T2 durante o mês de abril, devido à nitrificação do amônio a nitrito pelas bactérias do gênero Nitrossomonas.

Os valores de transparência, dispostos na Figura 6 (a), são menores que o encontrado por Moscoso et al. (1992), o qual variaram entre 18 a $20 \mathrm{~cm}$. Porém, próximo ao reportado por Felizatto (2000), a transparência foi de 12 e $11 \mathrm{~cm}$ para $\mathrm{T} 1$ e T2, respectivamente. Os valores obtidos, se comparados à piscicultura convencional, bem como a outros estudos que abordam o reúso em piscicultura, sugerem um ambiente com excesso de biomassa algal. De acordo com Ostrensky; Boeger (1998), esta alta concentração de biomassa de algas está associada, consequentemente, a problemas de falta de oxigênio na água prejudicando a sobrevivência dos peixes. Este fato foi evidenciado no presente estudo, como alternativa para ajustar os valores de acordo com o esperado em uma piscicultura, poderia promover-se a renovação da água.

Na resolução, é estabelecido o máximo para a turbidez de 100 UNT. Este limite estabelecido na resolução foi ultrapassado (Figura 6 (b)), no mês de julho (T1), devido à presença de sólidos em suspensão, como detritos orgânicos associados à presença de micro-organismos (CT e $\mathrm{CF}$ ), que também estiveram acima do limite da resolução (Tabela 4) durante este mês, e possivelmente, a presença de algas e plâncton. Enquanto nos meses de dezembro (T1), janeiro (T2) e março (T2), ultrapassaram o limite da resolução, devido à baixa vazão de abastecimento, o valo de acesso entre os tanques estava seco, o que tornou a água mais turva.

Os valores da C.E (Figura 7 (a)) estiveram abaixo dos reportados em artigos tanto sobre reúso em piscicultura quanto na piscicultura convencional, estes resultados sugerem uma acentuada produção primária, a qual pode ser utilizada como indicador indireto de disponibilidade de nutrientes (SIPAÚBATAVARES, 1994; BASTOS et al., 2003). Entretanto, devese também considerar as águas de origem (Tabela 3), que apresentaram baixa C.E.. Em ambos os tanques houve pouca variação da C.E., indicando assim pouca oscilação na concentração de sais nos mesmos.

A salinidade de ambos os tanques, conforme pode ser observado na Figura 7 (b) permite que sejam classificadas na resolução do CONAMA como águas doces, ou seja, com salinidade igual ou inferior a 0,5 $\%$, antes deste estudo acreditava-se que estas eram salobras por serem advindas de um poço tubular.

\subsection{Análises microbiológicas}

Os resultados dos CT sugerem que apenas no mês de julho no T1 (Tabela 4) se pode afirmar que estes coliformes eram em sua totalidade fecais. Durante os demais meses, os coliformes presentes em ambos os tanques estiveram associados à outra origem, que não fecal.

Na resolução, é estabelecido para os CF que, em $80 \%$ ou mais, de pelo menos seis amostras coletadas durante um ano, não deverá ser excedido o limite de $1.000 \mathrm{NMP} / 100 \mathrm{~mL}$. Os valores encontrados neste estudo permitem concluir que apenas o T2 está apto para reutilização em piscicultura, conforme pode ser observado na Tabela 4 (T1) e 5 (T2). Porém, de acordo com o estudo de Moscoso et al. (1992), para minimizar o nível de contaminação por $\mathrm{CF}$ a fim de que o pescado seja apto ao consumo humano, deve apresentar o nível máximo de $10^{-4} \mathrm{NMP} / 100 \mathrm{~mL}$ nas águas dos tanques aquícolas, enquanto o nível máximo no efluente deve ser $10^{-5} \mathrm{NMP} / 100 \mathrm{~mL}$, esperando uma redução de um ciclo logarítmico entre o afluente e o tanque de piscicultura. Sob tais condições, a qualidade sanitária dos peixes foi considerada boa, pois os demais parâmetros analisados estiveram abaixo daqueles considerados prejudiciais à saúde humana, por isso os peixes cultivados foram considerados aptos para o consumo humano direto. Entretanto, admite-se que estes valores são superiores ao recomendado na resolução do CONAMA, como também pela WHO (1989) que era vigente no período desta pesquisa. 


\subsection{Coletas de peixes}

De acordo com Odum; Barrett (2008), qualquer unidade que apresente uma comunidade de um determinado local interagindo com o ambiente físico através de um fluxo de energia que conduza a uma estrutura trófica, a uma diversidade biótica e ao ciclo de materiais caracteriza-se como um ecossistema. Este conceito aplica-se a ambos os tanques e, de acordo com esta definição, há um ecossistema em cada um deles, pois a presença de vários espécimes de peixes demonstra que existe uma cadeia desenvolvida nestes. Foram encontrados elementos de todos os níveis tróficos, os que formam a produção primária (fitoplanctôn, zooplanctôn e macrófitas aquáticas, principalmente de lemnáceas no decorrer do ano, entre outras), os consumidores primários como os lambaris e barrigudinhos, os consumidores secundários formados por carpas e mussuns e o topo de cadeia que são as traíras, além de moluscos e insetos, os quais, na fase larval, apresentam um papel mais relevante na cadeia de detritos (ESTEVES, 1998). A presença de um ecossistema aquático desenvolvido nestes permite sugerir a potencialidade de reúso destas águas.

\section{Conclusão}

De um modo geral, com as análises efetuadas, pode-se concluir que as condições em que se encontram as águas dos tanques de estabilização da UNIPAMPA, Campus Uruguaiana, estão de acordo com parâmetros preconizados pela resolução 537/05 do CONAMA, exceto para OD em ambos os tanques e os CF no T1. Desta maneira, as águas residuárias do T2 apresentam melhores condições, sugerindo a possibilidade de reúso em atividades piscícolas.

\section{Referências}

APHA - American Public Health Association; AWWA - American Water Works Association; WPCF - Water Pollution Control Federation. Métodos normalizados para el análisis de aguas potables y residuales. Madrid: Ediciones Dias de Santos, 1992. 1816 p.

ALBANEZ, J. R.; MATOS, A. T. Aquicultura. In: MACEDO, J. A. B. Águas \& águas. Belo Horizonte, MG: CRQ, 2007. p. 1097-1146.

BALDISSEROTTO, B. Fisiologia de peixes aplicada à piscicultura. Santa Maria, RS: UFSM, 2009. 352 p.

BASTOS, R.K.X.; PEREIRA, C.M.; PIVELLI, R.P.; LAPOLLI, F.R.; LANNA, E.A.T. Utilização de esgotos sanitários em piscicultura. In: BASTOS,
R.K.X. Utilização de esgotos tratados em irrigação, hidroponia e piscicultura. Rio de Janeiro,RJ: ABES, Rima, 2003. p.193-223.

BAUMGARTEN, M. G. Z.; ROCHA, J. M. B.; NIENCHESKI, L. F. H. Manual de análises em oceanografia química. Rio Grande, RS: FURG, 1996. $132 \mathrm{p}$.

BARTONE, C. Reutilizacion de aguas residuales en las lagunas de estabilizacion de San Juan de Miraflores. Repercusiones sanitarias, ambientales y socioeconomicas. Boletín de la Oficina Sanitaria Panamericana, v. 101, n. 5, p. 425-451, 1986.

CONAMA - Conselho Nacional do Meio Ambiente. Resolução normativa do CONAMA nº 357, de 17 de março de 2005. Disponível em: $<$ http://www.mma.gov.br/port/conama/legiabre. cfm?codlegi=459>. Acesso em: 12 maio 2013.

GARCIA, L.O.; COPATTI, C.E.; WACHHOLZ, F.; FILHO, W. P.; BALDISSEROTTO, B. Freshwater temperature in the state of Rio Grande do Sul, Southern Brazil, and its implication for fish culture. Neotropical Ichthyology, Porto Alegre, v. 6, n. 2, p. 275-281, 2008.

ESTEVES, F. A. Fundamentos de limnologia. Rio de Janeiro, RJ: Editora Interciência, 1998. 602 p.

FELIZATTO, M. R. Reúso de água em piscicultura no distrito federal: Potencial para pós-tratamento de águas residuárias associado à produção de pescado. 2000. 175 f. Dissertação (Mestrado em Tecnologia Ambiental e Recursos Hídricos) Universidade de Brasília, Brasília. 2000.

HESPANHOL, I. Potencial de Reúso de Água no Brasil: agricultura, indústria, município e recarga de aquíferos. In:MANCUSO, P. C. S.; SANTOS, H. F. Reúso de Água. São Paulo, SP: Manole, 2003. p.37-96.

KUBITZA, F. Qualidade da água na produção de peixes - Parte II. Panorama da Aquicultura, v. 8, n. 46, p. 35-41, 1998a.

KUBITZA, F. Qualidade da água na produção de peixes - Parte III. Panorama da Aquicultura, v. 8, n. 47, p. $35-43,1998 b$.

KUBITZA, F. Tilápia: tecnologia e planejamento na produção comercial. Jundiaí, SP: Editora Acqua \& Imagem, 2000. 285p.

LIMA, R. L; BRAUN, N.; KOCHHANN, D.; 
LAZZARI, R.; NETO, J.R.; MORAES, B.S.; LORO, V.L.; BALDISSEROTTO, B.Survival, growth and metabolic parameters of silver catfish, Rhamdia quelen, juveniles exposed to different waterborne nitrite levels. Neotropical Ichthyology, Porto Alegre, v. 9, n. 1, p. 147-152, 2011.

MONTENEGRO, L.A. Biologia alimentar e morfohistologia do tubo digestório do mussum, Synbranchus marmoratus, Bloch (Osteichthyes: Synbranchidae) no açude Marechal Dutra "gargalheiras", localizado no semi-árido brasileiro. 2009. 77 f. Dissertação (Mestrado em Ciências Biológicas) - Universidade Federal do Rio Grande do Norte, Natal. 2009.

MOSCOSO, J.; GALECIO, F. Reutilización de las aguas residuales para piscicultura - Revisión de literatura. Proyecto Evaluación y funcionamiento de las lagunas de San Juan. CEPIS - Centro Panamericano de Ingeniería Sanitaria y Ciencias del Ambiente. Lima, Peru, 1978.

MOSCOSO, J.; LEÓN, G.; MERINO, E.G.; MUÑOZ,A.F. Reuso en acuicultura de las aguas residuales tratadas en las lagunas de estabilización de San Juan. Sección II: Tratamento de las aguas residuales y aspectos sanitarios. CEPIS - Centro Panamericano de Ingeniería Sanitaria y Ciencias del Ambiente, Lima, Peru, 1992.

ODUM, E.P.; BARRETT, G. W. Fundamentos de Ecologia. São Paulo, SP: Cengage Learning Editora, 2008. 612 p.

OSTRENSKY, A.; BOEGER, W. Piscicultura: fundamentos e técnicas de manejo. Guaíba: Agropecuária, 1998. 211 p.

PEREIRA, L.P.F.; MERCANTE, C.T.J. A amônia nos sistemas de criação de peixes e seus efeitos sobre a qualidade da água: Uma revisão. Boletim do Instituto da Pesca, São Paulo, v. 31, n. 1. p. 81-88, 2005.

SANTOS, E. S. Cultivo do peixe ornamental molinésia como alternativa para o uso de esgoto tratado na piscicultura. 2012. $162 \mathrm{f}$. Tese (Doutorado em Engenharia Civil) - Universidade Federal do Ceará, Fortaleza. 2012.

SIPAÚBA-TAVARES, L.H. Limnologia aplicada à aquicultura. Jaboticabal, SP: FUNEP, 1994. 70 p.

TAVARES, F.A. Reúso de água e polimento de efluentes de lagoas de estabilização por meio de cultivo consorciado de plantas da família lemnaceae e tilápias. 2008. 237 f. Tese (Doutorado em Engenharia ambiental) - Universidade Federal da Santa Catarina, Florianópolis. 2008.

VON SPERLING, M. Introdução à qualidade da água e ao tratamento de esgotos. Belo Horizonte, MG: UFMG, 2005. 243 p.

WHO - World Health Organization. Health guidelines for the use of wastewater in agriculture and aquaculture. Genebra: Technical Report Series, $1989.75 \mathrm{p}$. 